\title{
Design of Data Acquisition System for Engine Experiment
}

\author{
Xinli Wang ${ }^{1, \mathrm{a}^{*}}$, Ping $\mathrm{Xu}^{2, \mathrm{~b}}$, Jianping $\mathrm{Bi}^{1, \mathrm{c}}$ \\ ${ }^{1}$ Department of Mechanical Engineering, Henan Institute of Engineering, Zhengzhou, 451191 China \\ ${ }^{2}$ College of Civil Engineering, Henan Institute of Engineering, Zhengzhou, 451191 China \\ a wangxinli001@sina.com, bxping_226111@163.com,c bijianping77@yahoo.com.cn
}

Keywords: Data Acquisition; Engine Experiment; Hardware Design; Software Design

\begin{abstract}
With the development of computer technology, data acquisition can reduce the heavy manual recording and ensure accurate and effective experiments. Engine experiment data acquisition system is designed in this paper. First the system hardware is completed and the sensor processing circuit is done based on different sensors. Then the software for the engine data acquisition system is achieved based on $\mathrm{VC}++$ platform.
\end{abstract}

\section{Introduction}

With the development of computer technology in all areas, real-time data acquisition, data display, real-time control are possible during the experiment, which can reduce the heavy manual recording and ensure accurate and effective experiments.

In the 1950s, the data acquisition device began to appear. US-developed test system for data acquisition is the symbol and the system is mainly used in the military field. This kind of data acquisition device may be operated by unfamiliar person. Around the late 1960s, data acquisition products appeared in foreign markets, and these data acquisition products were used in more specific areas. In the late 1970s, with the development of micro-computer, data acquisition and control computer equipment acquisition integration began. In the 1980s, with the popularity of computers, the universal data acquisition device began to appear [1]. During this period, the data collection device is mainly composed by the acquisition, interface bus and control computer and the interface bus used almost based on GPIB. In the late 1980s, the data acquisition device consisted of IPC, microcontroller and integrated circuits. Also, the hardware was replaced by the software, which would reduce the cost and size and increase the performance. After the 1990s, in developed countries, the data acquisition technology has been widely used in military, aerospace and industrial f fields. With the development of integrated circuit technology, there has been a high-performance single-chip data acquisition system (DAS). DAS resolution is up to 16, and the sampling rate is up to hundreds of thousands of times per second. Therefore, the data acquisition technology has become a technology. During this period, the data collection devices modular structure is divided into parallel bus interface bus and serial bus. Parallel interface bus is represented by VXI bus and PXI for local data acquisition, which is mostly used in the military field. Recent data collected in the field is undergoing significant changes. First, the distributed control applications of intelligent data acquisition system are being developed. Secondly, the number of personal computer data acquisition system is also increasing.

However, duo to the prices and some specialized systems, it is not suitable for all data acquisition system [2]. Then one data acquisition system based on VC++ platform and single chip MCU is designed in this paper.

\section{Hardware}

The system hardware is divided into data acquisition, real-time signal acquisition module and data processing module. It can be seen in Fig. 1. 
The data acquisition includes the sensor and communication system. And the real-time signal acquisition module is the PCI data acquisition system. Then the data processing module is the computer.

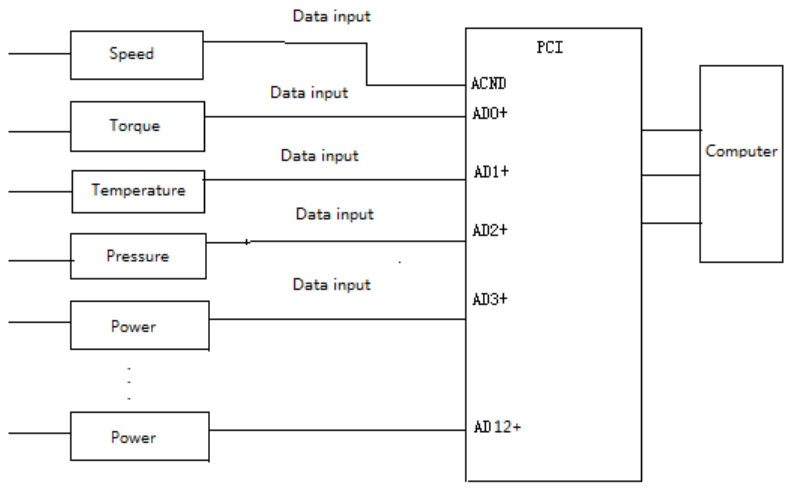

Fig. 1 Diagram of the system

\section{Sensor Processing}

The sensor signal can be divided into analog and digital signals. For the designed control system, most of the sensor signals are analog signals, which can be roughly classified into the linear signals and the sine signals.

Sine Signal Processing. Judge the dead position and the engine speed obtained by magnetic sensors according to the output characteristics of the sensors, the output analog signal is sine signal, as shown in Fig. 2.

Since the position between the fixed top dead center and the magnetic sensors is fixed, it can be used to determine the TDC and it also can be used to calculate the speed [3,4]. In addition, the first thing is to convert the magnetic signals into TTL signal. Its processing circuit is shown in Fig. 3, where the 6N137 is used as digital switching and the sine signal is converted into high or low digital signal (Fig. 4). Then the signal can be directly input microcontroller by utilizing I/ O port [5].

Note that when using this method for signal processing the circuit, there will be error because it takes some time for $6 \mathrm{~N} 137$ to transmit.
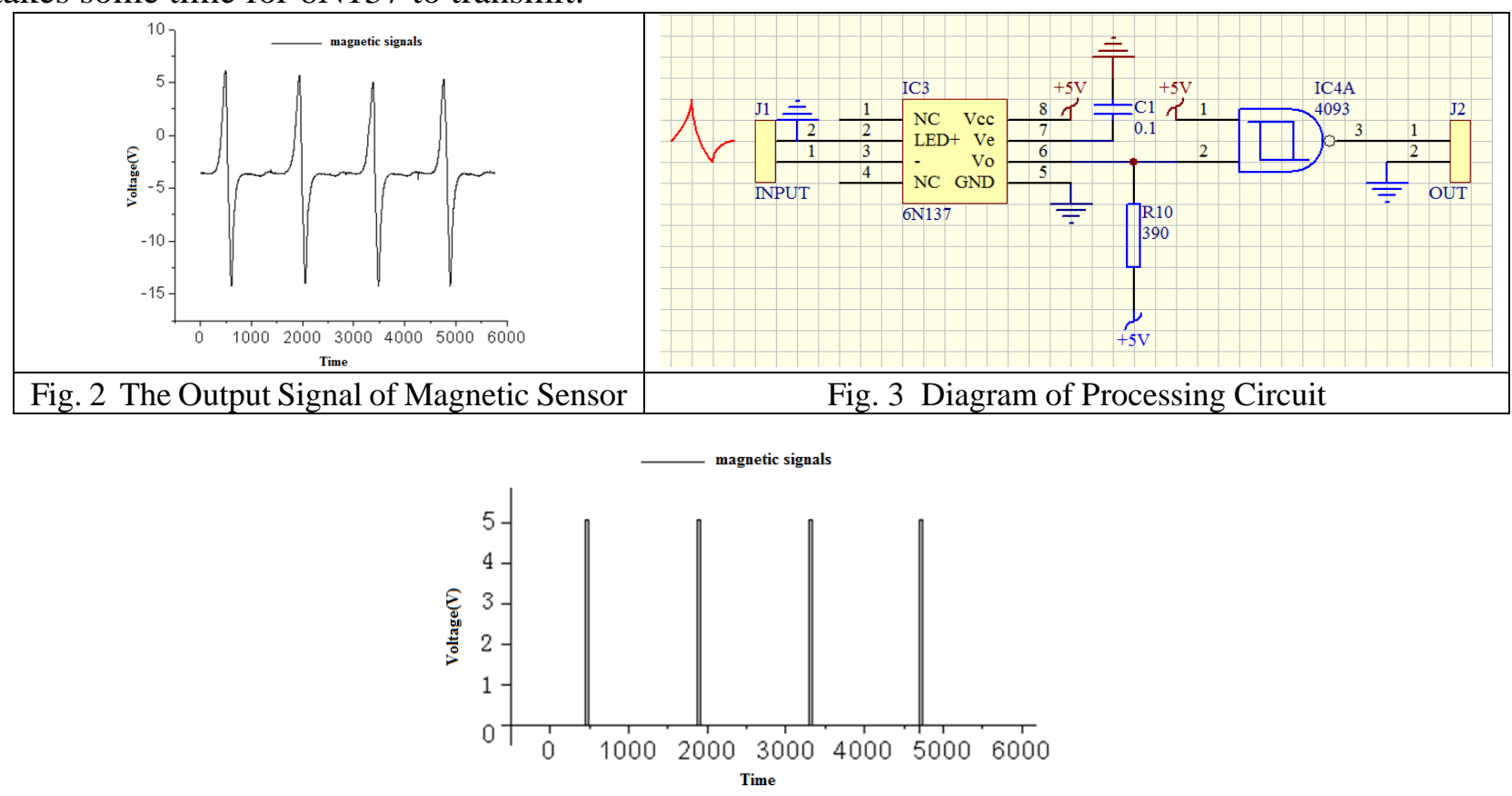

Fig. 4 Signal Conversion 
Linear Signal Processing. If the output signal of the analog signal is voltage value and there is a linear relationship between the measured signal and the output signal, it can be input the ECU by A/D converter [6]. However, if the amplitude of the analog signal is small, it cannot make full use of A/D conversion precision, and the signal requires to be enlarged; if the amplitude is large, the limit processing is required before inputting the ECU. For this control system, the throttle position sensor signal and the intake pressure sensor output are a linear curve, as shown in Fig. 5. The output signal is a voltage signal between $0 \sim 5 \mathrm{~V}$, and the amplitude range is within the microcontroller capacity and it can be input directly to $\mathrm{A} / \mathrm{D}$ ports.
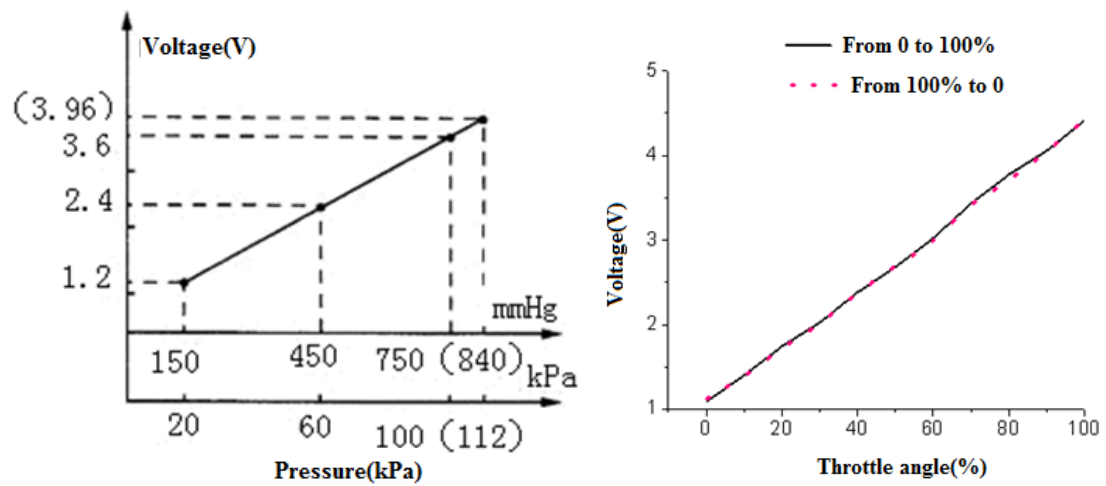

(a) Intake Air Pressure (b) Throttle Vale Angle

Fig. 5 Liner Signals

\section{Software Design}

In this paper, the programming process is emphasized and it can be achieved through two stages, including the basic interface design and coding.

Basic Interface Design. The basic interface design bases on the development of interface establishment, add of basic controller and design of display interface. The basic controllers include text controller, edit controller, button controller and Com-box controller [7, 8].

For text controller, its function is data display and label display. It is used to show input parameters and display output. For edit controller, it can realize the operation of enter, copy, cut, paste and delete and it is used to input initial data. The button controller is also common for interface design, and it has the program calculation ability.

The Com-box controller combines the features of edit box and list box, which can be used for data input and selecting part of a list box, and it is used to display different curve. The picture controller is used to show pictures, including bitmaps, icons, boxes, and metafiles that is not used for data input.

The basic interface is divided into three parts: the first is data input, the second is monitor and the third is data acquisition.

In the parameter input part, as shown in Fig. 6, the basic parameters include the engine parameters, experiment time, experiment place, experiment temperature and path of storage. Monitor part, as shown in Fig. 6, can check the value of different channel by using the curve display. Data acquisition can store the data to the computer.

Coding. The main program includes the data acquisition and data storage, which are designed based on $\mathrm{VC}++[9,10]$. And the data acquisition coding is shown in the following.

void CDiagnoserView::OnConfirm()

\{

UpdateData(true);

biCHselect $=0$;

bFirstCH=0;

for (short $\mathrm{i}=0 ; \mathrm{i}<6 ; \mathrm{i}++)$ 


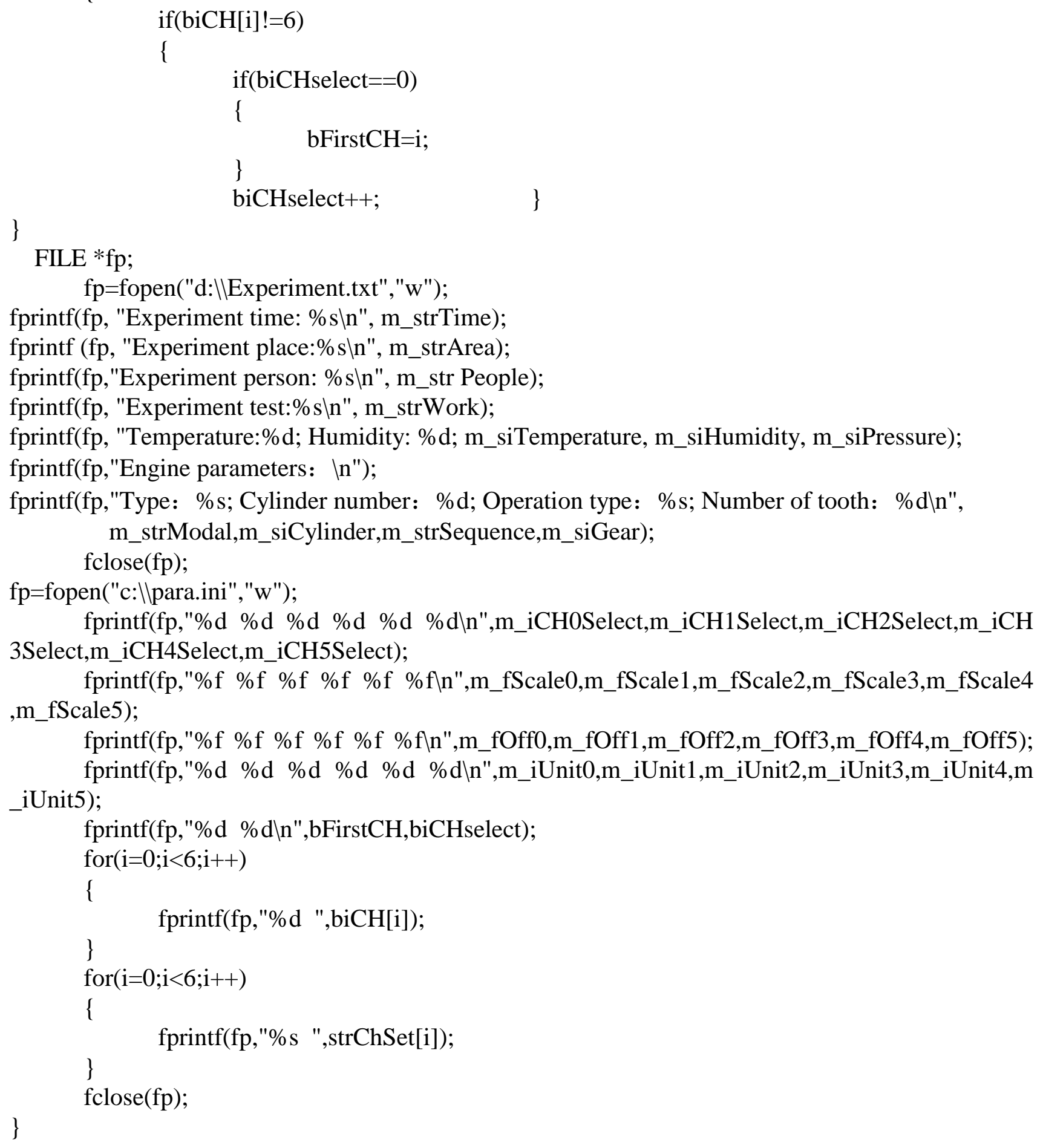




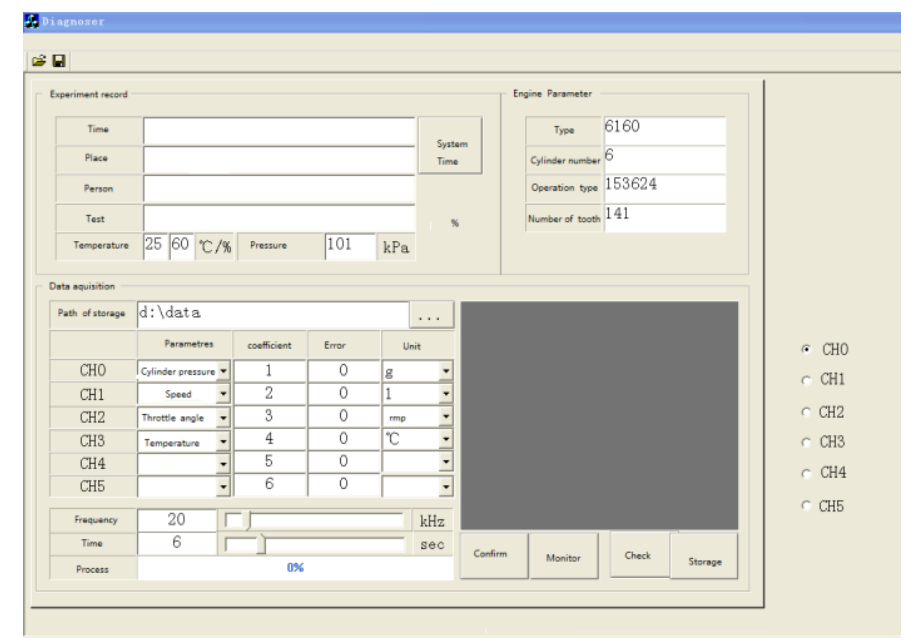

Fig. 6 Main interface of the system

\section{Summary}

In this paper, a data acquisition system for engine experiment is designed, including the hardware and software. The design of the controller hardware system is completed firstly, and then the sensor processing is improved. Meanwhile, the coding of the system has been designed.

\section{Acknowledgment}

The authors would like to express appreciation of financial support by the Key Subject of Machine Design and Theory, the Reliability Test Center and Modern Design Method Research Center of Henan Institute of Engineering.

\section{References}

[1] Xu Yong Ping, Cao zi yuan, Xu de bing. The Development of Management and Acquisition Software in the High Speed Data Acquisition System, 2nd International Symposium on Instrumentation Science and Technology, 2002, (01): 367-369.

[2] Geng Yubo, Zhang Xiaodong. A Data Acquisition System Based on PCI Bus for the Digital Nuclear Magnetic Resonance Image Spectrometer, The Seventh International Conference on Electronic Measurement and Instruments, 2005, (06): 89-91.

[3] D. Li-tao, L. Rong-chang, W. Qing-zhu, et al. Crankshaft Crankshaft Strength and Fatigue Strength of Research Methods to Improve Life, Tractor \& Farm Transporter, 2004 (05) :18-20.

[4] K. Jun-feng. 1.3L Gasoline Engine Crankshaft Strength Analysis of Car, Shanghai: Shanghai Communications University, 2007

[5] H. Song-tao, H. Zhi-yong. Mode Analysis of Three dimensional Finite Element and Experimental Study on a 6102B Diesel Engine Crankshaft, Transactions of The Chinese Society of Agricultural Machinery, 2001(32): 74-77

[6] W. Yong-lian. Mechanics of Materials, Beijing: Mechanical Industry Press, 2009

[7] S. Kun, L. Rui-ning. Visual C + + Programming Self-study Manual, People Post Press, 2008

[8] Pan Xufeng. Modern Automotive Electronic Technology. Beijing: Beijing Institute of Technology Press, 1998. 
[9] WANG Sui. Double Principle and Maintenance: Automotive Electronic Control Systems. Beijing: Beijing Institute of Technology Press, 1995.

[10]Wang Yu. Study of Low Calorific Value Gaseous Fuel Engine Performance, Beijing: Beijing Jiaotong University, 2008 